\title{
EDITORIAL \\ COVID-19 in children and altered inflammatory responses
}

\author{
Pediatric Research (2020) 88:340-341; https://doi.org/10.1038/s41390- \\ 020-0881-y
}

\section{BACKGROUND}

The coronavirus disease (COVID-19) pandemic while affecting all age groups appears to be less severe in children. In this journal, we have international contributions relaying experiences from paediatricians across the world. Patients aged $<18$ years only account for $2 \%$ of severely affected patients. However, children can still be vectors if they are asymptomatic and shedding the virus. Dong et al. described a cohort of 2143 children with suspected infection. In 34\%, infection was confirmed but there was little critical illness and one death. There appeared to be proportionally more severe illness in infants, a result that could have be confounded by concomitant bronchiolitis. ${ }^{1}$ Chen et al. and others have also shown that there is no evidence of vertical transmission in pregnant women, ${ }^{2-5}$ although this possibility may still exist. Possible reason for this disparity in severity between adults and children may relate to differences in receptors in the renin-angiotensin system (RAS) and altered inflammatory responses to pathogens.

\section{ANGIOTENSIN-CONVERTING ENZYME 2 (ACE2) RECEPTOR}

In this issue of the journal, Zhu et al. have looked for reasons that children have relatively milder illness. ${ }^{6}$ Alterations in immune function and crucial receptors in the RAS are implicated. The virus uses the ACE2 receptor to invade cells, which is the same receptor as that for severe acute respiratory syndrome coronavirus (SARS-CoV), and mainly spreads through the respiratory tract. These receptors are present on many cell types in the body including immune cell such as monocytes, neutrophils and lymphocytes. The RAS system is associated with inflammation via angiotensin II, and ACE2 alters RAS activity from proinflammatory to anti-inflammatory responses. ${ }^{7}$ Specific inhibitors such as angiotensin II type 1 receptor (AT1R) antagonist, losartan, have been shown to be effective in animal models of septic shock. ${ }^{8}$ Patients with either very high ACE2 levels (such as in diabetes or cardiovascular disease) or very low levels (animal models of hypertension) can have an abnormal immune response and pulmonary inflammation. ${ }^{9}$ Decreasing ACE2 is notable in animal models of ageing. ${ }^{10}$ Sodhi et al. demonstrated that neutrophil inflammation following bacterial pneumonia was altered by pulmonary ACE2 activity. Alterations in ACE2 are critical for neutrophil influx and lung inflammation. ${ }^{9}$ In respiratory syncytial virus (RSV), ACE2 protected against severe lung injury both in children and an experimental mouse model. RSV disease pathogenesis was worsened via activation of AT1R, and a recombinant ACE2 decreased the severity of RSVassociated lung injury (Guo et al., scientific reports). ${ }^{8}$ However, Schouten et al. found that in contrast to preclinical models there are no significant differences in ACE and ACE2 in different age groups from newborns to old age. ${ }^{11,12}$

\section{INFLAMMATION}

Inflammatory responses in adults and children differ and vary throughout the lifespan. ${ }^{13}$ Schouten et al. found that increasing proinflammatory cytokines associated with neutrophil function with age also correlated with severity of acute respiratory distress syndrome (ARDS) and may partially explain age-dependent difference. ${ }^{11}$ Levels of myeloperoxidase, interleukin (IL)-6, IL-10 and p-selectin were higher with increasing age, whereas intercellular adhesion molecule-1 was higher in neonates in bronchoaveolar lavage samples. Wong et al. found that 2360 genes in neutrophils, 965 in monocytes and 109 genes in lymphocytes were upregulated or downregulated in paediatric septic shock, reinforcing the data that circulating lymphocytes are not the main leukocyte population with altered gene profiles during septic shock. ${ }^{14}$ Wynn et al. found dramatic differences in the transcriptomic response related to age in paediatric septic shock. ${ }^{15}$ Jeljeli et al. studied the ontogeny of cytokine production in response to phytohaemagglutinin from neonates to adults and noted the change from increased IL-10 as neonates to balanced IL-10/T helper type 1 (Th1)/Th2/Th17 cytokine levels early in life. This allows protection from pathogens but ameliorates the cytokine storm. ${ }^{16}$

Severe COVID-19 infection is characterised by a massive proinflammatory response or cytokine storm that results in ARDS and multiorgan dysfunction. This result suggests that the aetiology may be haemophagocytosis or macrophage activation syndrome. It is suggested that patients with severe COVID-19 should be screened for hyperinflammation using laboratory trends (e.g. increasing ferritin, decreasing platelet counts or erythrocyte sedimentation rate) to identify the subgroup of patients for whom anti-inflammatory treatment could improve mortality. ${ }^{1}$

Therapeutic options include steroids, intravenous immunoglobulin, selective cytokine blockade (e.g. anakinra or tocilizumab), Remdesivir, hydoxyxhrolquine and Janus kinase inhibition. ${ }^{17}$ Remdesivir (GS-5734) blocks RNA-dependent polymerase and is a nucleotide analogue prodrug currently in clinical trials for treating Ebola virus infections. It has a broad spectrum of antiviral activities against RNA viruses, including SARS-CoV and Middle East respiratory syndrome $\mathrm{CoV}{ }^{18}$ Chloroquine blocks viral entry into endosomes. Hydroxychloroquine in combination with azithromycin showed promise in an open-label, non-randomised clinical trial of treatment in COVID-19. ${ }^{8}$ Chloroquine appeared to limit the replication of SARS-CoV-2 (virus causing COVID-19) in vitro in a systematic review of six studies. ${ }^{19}$

There is an ongoing clinical trial in China including severely affected patients with elevated IL- 6 that have been treated with tocilizumab. This study has had positive results and shown improvement in symptoms in 20 patients enrolled to date (refer COVID-19 pneumonia and elevated IL-6 in China (ChiCTR2000029765)). ${ }^{20}$ Tocilizumab targets the IL-6 receptor and is a monoclonal antibody. It is approved for use in giant cell arteritis and rheumatoid arthritis. However, in disorders such as juvenile immune arthritis it appears that IL- 6 blockade alone is insufficient to control the inflammatory cascade, especially in patients who are afebrile with lower cell counts and ferritin levels, and higher liver enzymes. ${ }^{21}$ Corticosteroids block inflammation but the current interim World Health Organisation guidance advised against their use due to the lack of evidence for benefit 
and the risks of harm due to immunosuppression and secondary bacterial or fungal infection. ${ }^{22}$

Sepsis has been described as two different phases as the body responds to systemic infection. First, there is a profound initial proinflammatory phase or cytokine storm. This is then followed by a period of potentially prolonged immunosuppression. ${ }^{23}$ This dysregulated immunosuppression phase is the major cause of sepsis-related fatalities. The immune suppression and dysregulation associated with sepsis is undeniably the major cause of sepsis-related fatalities. ${ }^{24}$ Therefore, anti-inflammatory therapies administered in this phase would be deleterious. This suggests that further information on the individualised immune response would be useful to initiate therapies such as anti-IL-6, and monitoring of proinflammatory and anti-inflammatory responses throughout the treatment course would be valuable. ${ }^{25}$ In addition, sepsis definitions and responses are known to be different in children and neonates. ${ }^{26}$ Thus there are several reasons why children may fare better if infected with COVID-19. In addition, further understanding the differences in immune responses in difference age groups is valuable for targeted immunotherapies.

\section{ACKNOWLEDGEMENTS}

This work was supported by the National Children's Research Centre, Crumlin, Dublin, Ireland and Health Research Board Ireland.

\section{ADDITIONAL INFORMATION}

Competing interests: The authors declare no competing interests.

Publisher's note Springer Nature remains neutral with regard to jurisdictional claims in published maps and institutional affiliations.

Eleanor J. Molloy (D) ${ }^{1,2,3,4,5}$ and Cynthia F. Bearer ${ }^{6,7}$ ${ }^{1}$ Discipline of Paediatrics, Trinity College, the University of Dublin, Dublin, Ireland; ${ }^{2}$ Children's Health Hospital (CHI) at Tallaght, Tallaght University Hospital, Dublin, Ireland; ${ }^{3}$ Trinity Translational Medicine Institute, St James Hospital, Dublin, Ireland; ${ }^{4}$ Neonatology, $\mathrm{CHI}$ at Crumlin, Dublin, Ireland; ${ }^{5}$ Paediatrics, Coombe Women's and Infant's University Hospital, Dublin, Ireland; ${ }^{6}$ Division of Neonatology, Department of Pediatrics, Rainbow Babies \& Children's Hospital, Cleveland, $\mathrm{OH}$, USA and ${ }^{7}$ Case Western Reserve University School of Medicine, Cleveland, $\mathrm{OH}$, USA Correspondence: Eleanor J. Molloy (eleanor.molloy@tcd.ie)

\section{REFERENCES}

1. Dong, Y. et al. Epidemiological characteristics of 2143 pediatric patients with 2019 coronavirus disease in China. Pediatrics https://doi.org/10.1542/peds.20200702 (2020).

2. Chen, $\mathrm{H}$. et al. Clinical characteristics and intrauterine vertical transmission potential of COVID-19 infection in nine pregnant women: a retrospective review of medical records. Lancet 395, 809-815 (2020).

3. Chen, Y. et al. Infants born to mothers with a new coronavirus (COVID-19). Front. Pediatr. 8, 104 (2020).

4. Li N, Han L, Peng M, et al. Maternal and neonatal outcomes of pregnant women with COVID-19 pneumonia: a case-control study [published online ahead of print, 2020 Mar 30]. Clin. Infect. Dis. ciaa352, https://doi.org/10.1093/cid/ciaa352 (2020).
5. Schwartz, D. A. An analysis of 38 pregnant women with COVID-19, their newborn infants, and maternal-fetal transmission of SARS-CoV-2: maternal coronavirus infections and pregnancy outcomes. Arch. Pathol. Lab. Med. https://doi.org/ 10.5858/arpa.2020-0901-SA (2020).

6. Zhu, L., Lu, X. \& Chen, L. Possible causes for decreased susceptibility of children to coronavirus. Pediatr. Res. https://doi.org/10.1038/s41390-020-0892-8 (2020).

7. Rodrigues Prestes, T. R., Rocha, N. P., Miranda, A. S., Teixeira, A. L. \& Simoes-E-Silva, A. C. The anti-inflammatory potential of ACE2/angiotensin-(1-7)/Mas receptor axis: evidence from basic and clinical research. Curr. Drug Targets 18, 1301-1313 (2017).

8. Guo, J. et al. Effects of angiotensin II type 1 receptor antagonist on rats with septic shock. Int. J. Clin. Exp. Med. 8, 7867-7871 (2015).

9. Sodhi, C. P. et al. A dynamic variation of pulmonary ACE2 is required to modulate neutrophilic inflammation in response to Pseudomonas aeruginosa lung infection in mice. J. Immunol. 203, 3000-3012 (2019).

10. Yoon, H. E. et al. Age-associated changes in the vascular renin-angiotensin system in mice. Oxid. Med. Cell Longev. 2016, 6731093 (2016).

11. Schouten, L. R. et al. Age-dependent differences in pulmonary host responses in ARDS: a prospective observational cohort study. Ann. Intensive Care 9, 55 (2019).

12. Olin, A. et al. Stereotypic immune system development in newborn children. Cell 174, 1277-1292.e14 (2018)

13. Wong, H. R., Freishtat, R. J., Monaco, M., Odoms, K. \& Shanley, T. P. Leukocyte subset-derived genome-wide expression profiles in pediatric septic shock. Pediatr. Crit. Care Med. 11, 349-355 (2010).

14. Wynn, J. L. et al. The influence of developmental age on the early transcriptomic response of children with septic shock. Mol. Med. 17, 1146-1156 (2011).

15. Jeljeli, M. et al. Ontogeny of cytokine responses to PHA from birth to adulthood. Pediatr. Res. 86, 63-70 (2019).

16. Mehta, P. et al. COVID-19: consider cytokine storm syndromes and immunosuppression. Lancet https://doi.org/10.1016/S0140-6736(20)30628-0 (2020).

17. Martinez, M. A. Compounds with therapeutic potential against novel respiratory 2019 coronavirus. Antimicrob. Agents Chemother. https://doi.org/10.1128/ AAC.00399-20 (2020).

18. Gautret et al. An in vitro Shwartzman reaction-like response is augmented agedependently in human peripheral blood mononuclear cells. J. Leukoc. Biol. 79, 463-472 (2006).

19. Cortegiani, A., Ingoglia, G., Ippolito, M., Giarratano, A. \& Einav, S. A systematic review on the efficacy and safety of chloroquine for the treatment of COVID-19. J. Crit. Care https://doi.org/10.1016/j.jcrc.2020.03.005 (2020).

20. Chinese Clinical Trial Registry. A multicenter, randomized controlled trial for the efficacy and safety of tocilizumab in the treatment of new coronavirus pneumonia (COVID-19). http://www.chictr.org.cn/showprojen.aspx (2020).

21. Irabu, H. et al. Comparison of serum biomarkers for the diagnosis of macrophage activation syndrome complicating systemic juvenile idiopathic arthritis during tocilizumab therapy. Pediatr. Res. https://doi.org/10.1038/s41390-020-0843-4 (2020).

22. WHO. Clinical management of severe acute respiratory infection when nove coronavirus (nCoV) infection is suspected. https://www.who.int/publicationsdetail/clinical-management-of-severe-acute-respiratory-infection-when-novelcoronavirus-(ncov)-infection-is-suspected (2020).

23. Hotchkiss, R. S., Monneret, G. \& Payen, D. Sepsis-induced immunosuppression: from cellular dysfunctions to immunotherapy. Nat. Rev. Immunol. 13, 862-874 (2013).

24. Daviaud, F. et al. Timing and causes of death in septic shock. Ann. Intensive Care 5, 16 (2015).

25. Ritchie, A. I. \& Singanayagam, A. Immunosuppression for hyperinflammation in COVID-19: a double-edged sword? Lancet https://doi.org/10.1016/S0140-6736(20) 30691-7 (2020).

26. Molloy, E. J. et al. Neonatal sepsis: need for consensus definition, collaboration and core outcomes. Pediatr. Res. https://doi.org/10.1038/s41390-020-0850-5 (2020). 